\title{
URINE PRECIPITIN REACTION FOR SCHISTOSOMIASIS JAPONICA
}

\author{
KOYO OKABE AND TAKAHUMI TANAKA \\ Department of Parasitology, Kurume University School of Medicine, \\ Kurume-shi, Japan
}

\section{INTRODUCTION}

In cases of helminthiasis caused by an alimentary canal parasite, such as Ascaris or hookworm, the fecal examination is sufficient for its diagnosis. In cases of helminthiasis caused by a tissue parasite, however, the fecal examination cannot be sufficient, as the eggs of the parasite are not always discharged regularly in feces. In acute schistosomiasis japonica it takes about one month to discover the ova, and in chronic cases we cannot always find the eggs in the feces. Accordingly, it is very difficult to evaluate the therapeutic effects of medicine for schistosomiasis. It is not seldom that some patients discharge the eggs in several months after the treatment. Many concentration methods have been studied for the Schistosoma eggs, and yet these methods are not perfect. Immunological examinations such as the intradermal test, the precipitin test, the flocculation test or the complement fixation test have been investigated with the ova, cercariae, and the adult antigens by many authors. Okabe et al. (1954) reported that the precipitin reaction with the adult antigens was positive for acute schistosomiasis japonica with dilution as high as $1: 6400$, but the reaction was lower in chronic cases, only with the dilution of $1: 800$. Matsuse (1956) in our department reported that the precipitin reaction became positive in rabbits after 4 weeks from the exposure to cercariae.

It is probable that metabolic products of the worms in the blood are discharged sooner or later through the kidney into the urine. The authors intended to catch the metabolic products in the urine by the precipitine reaction and reported the obtained results in the preliminary report (1958). The urine precipitin reaction was examined in experimental animals for the evaluation of the therapeutic effects and for the earlier diagnosis of schistosomiasis japonica.

\section{MATERIALS ANDiMETHODS}

\section{Materials}

a. The urine of schistosomiasis patients

The urine discharged early in the morning from a schistosomiasis patient was condensed up to one third with a vacuum pump at $40^{\circ} \mathrm{C}$ and dialysed with cellophane in running water for 24 hours. In summer, when the urine was left by for several days before the concentration, sodium azide was added to it at a dilution of $0.1 \%$. The condensed urine was diluted with saline solution in a series of twofold dilutions. 


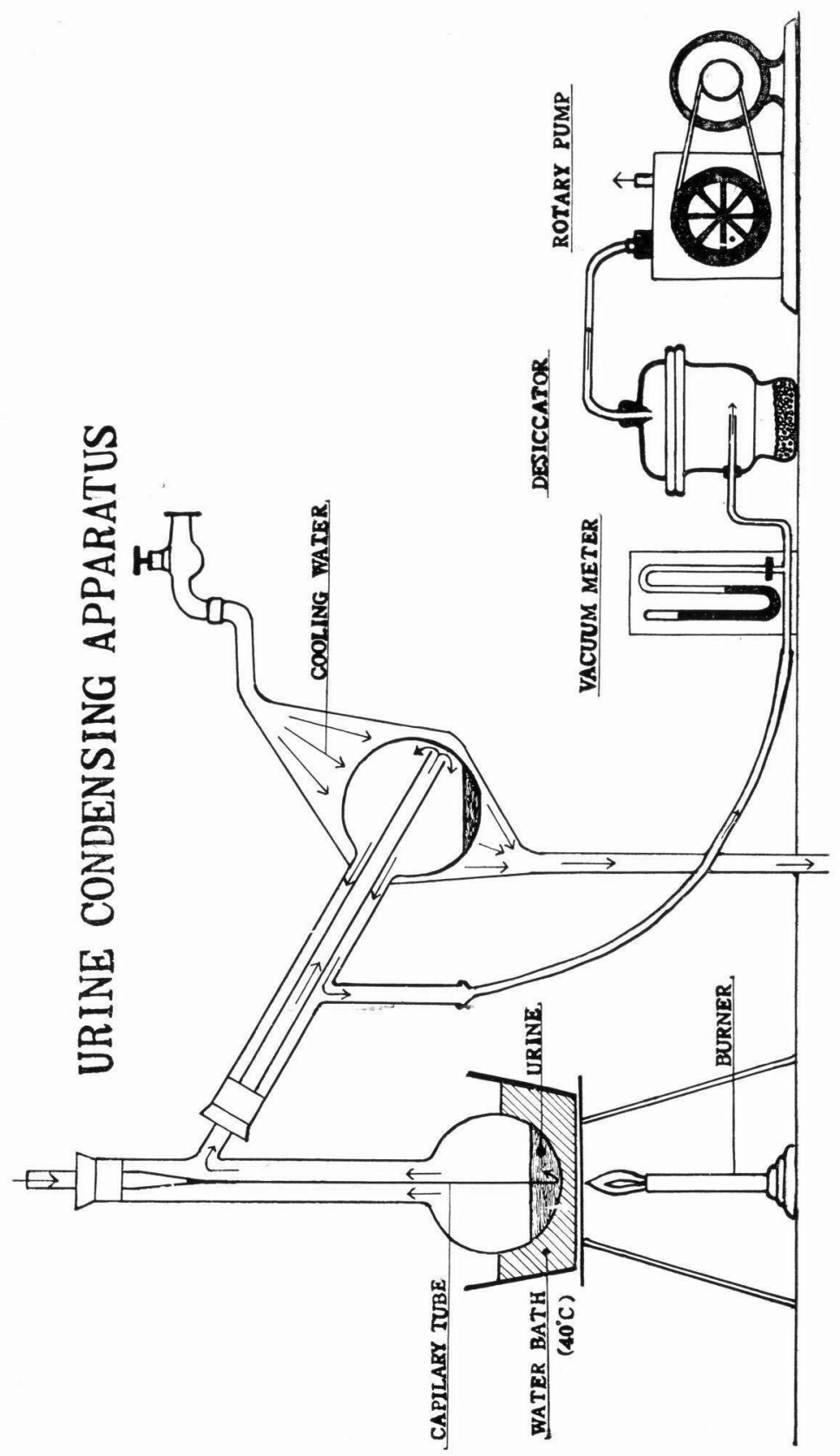


b. The urine of schistosome infected rabbits.

The urine was catheterized and condensed up to one third, dialysed for 24 hours, and used as an antigen.

c. Serum of rabbits immunized with the schistosome antigen.

Schistosoma japonicum removed from rabbits were washed with saline solution and dried in a dessicator. Dried worms were put in Coca's solution ( $0.5 \%$ $\mathrm{NaCl}, 0.5 \% \mathrm{NaHCO}_{3}$ and $0.4 \%$ phenol) at a dilution of $1: 100$, stored in an icebox at $4^{\circ} \mathrm{C}$ for 24 hours, and extracted. Five $\mathrm{ml}$ of the antigen was injected intravenously into a rabbit, seven times every two days. After the last injection the blood was removed when the serum was over a titer of 1:3200. The immune serum was stored in a deepfreeze at $-20^{\circ} \mathrm{C}$ and the amount desired was thawed for use.

2. Methods

The serum was divided into small precipitin tubes and the urine antigens which had been obtained by diluting with saline solution in a series of twofold dilutions (See II-1-a) were overlayed.

Readings were made after 2 hours. (In summer the temperature of the place where the test was incubated was $26^{\circ} \mathrm{C}$.) The degree of white ring formation at the interphase was the measure of reading the reaction.

\section{RESULTS}

\section{Acute cases}

The urine precipitin reaction was tried and examined on two acute cases. These patients had a chill and fever. Liver tumor, eosinophilia and an increase of red cell sedimentation rate were also observed on them. A fecal examination was not done.

The titer of one of them was $1: 4$ and the other's $1: 2$, while the serum precipitin test showed the $1: 800$ titer in each case.

TABLE 1

The urine precipitin reaction for acute schistosomiasis japonica patients

\begin{tabular}{c|c|c|c|c|c|c|c|c|c}
\hline Name & Age & Sex & $1: 1$ & $1: 2$ & $1: 4$ & $1: 8$ & $1: 16$ & Cont. & $\begin{array}{c}\text { Serum precipitin } \\
\text { reaction }\end{array}$ \\
\hline K. N. & 12 & $\mathrm{~m}$ & + & + & + & - & - & - & $1: 800$ \\
K. K. & 66 & $\mathrm{~m}$ & + & + & - & - & - & - & $1: 800$ \\
\hline
\end{tabular}

2. Sub-acute cases

Two sub-acute cases were examined. Both of them complained of a malaise and had a little fever. A fecal examination was not done.

The titer of the one was $1: 2$ and the other's $1: 4$.

TABLE 2

The urine precipitin reaction for sub-acute schistosomiasis japonica patients

\begin{tabular}{l|c|c|c|c|c|c|c|c|c}
\hline Name & Age & Sex & $1: 1$ & $1: 2$ & $1: 4$ & $1: 8$ & $1: 16$ & Cont. & $\begin{array}{c}\text { Serum precipitin } \\
\text { reaction }\end{array}$ \\
\hline M. I. & 12 & $\mathrm{~m}$ & + & + & - & - & - & - & $1: 400$ \\
K. N. & 8 & $\mathrm{~m}$ & + & + & + & - & - & - & $1: 800$ \\
\hline
\end{tabular}




\section{Chronic cases}

102 cases in whose feces schistosome eggs were found by mass-examination were examined. All cases turned out to be positive for the urine precipitin reaction.

TABLE 3

The urine precifitin reaction for chronic schistosomiasis japonica patients

\begin{tabular}{|c|c|c|c|c|c|c|c|c|c|c|}
\hline No. & Name & Age & Sex & $1: 1$ & $1: 2$ & $1: 4$ & $1: 8$ & $1: 16$ & Cont. & $\begin{array}{l}\text { Serum precipitin } \\
\text { reaction }\end{array}$ \\
\hline $\begin{array}{l}1 \\
2 \\
3 \\
4 \\
5\end{array}$ & $\begin{array}{l}\text { M. O. } \\
\text { T. H. } \\
\text { M. O. } \\
\text { S. S. } \\
\text { H. S. }\end{array}$ & $\begin{array}{l}35 \\
48 \\
38 \\
46 \\
19\end{array}$ & $\begin{array}{l}\mathrm{m} \\
\mathrm{m} \\
\mathrm{m} \\
\mathrm{m} \\
\mathrm{m}\end{array}$ & $\begin{array}{l}+ \\
+ \\
+ \\
+ \\
+\end{array}$ & $\begin{array}{l}+ \\
+ \\
+ \\
+ \\
+\end{array}$ & $\begin{array}{l}- \\
+ \\
+ \\
+ \\
+\end{array}$ & $\begin{array}{l}- \\
- \\
- \\
+ \\
+\end{array}$ & $\begin{array}{l}- \\
- \\
+ \\
-\end{array}$ & $\begin{array}{l}- \\
- \\
- \\
-\end{array}$ & $\begin{array}{l}1: 1600 \\
1: 1600 \\
1: 1600 \\
1: 800 \\
1: 1600\end{array}$ \\
\hline $\begin{array}{r}6 \\
7 \\
8 \\
9 \\
10\end{array}$ & $\begin{array}{l}\text { M. A. } \\
\text { T. A. } \\
\text { N. M. } \\
\text { N. M. } \\
\text { T. M. }\end{array}$ & $\begin{array}{l}33 \\
39 \\
53 \\
31 \\
22\end{array}$ & $\begin{array}{l}\mathrm{m} \\
\mathrm{m} \\
\mathrm{m} \\
\mathrm{m} \\
\mathrm{m}\end{array}$ & $\begin{array}{l}+ \\
+ \\
+ \\
+ \\
+\end{array}$ & $\begin{array}{l}+ \\
+ \\
+ \\
+ \\
+\end{array}$ & $\begin{array}{l}- \\
+ \\
+ \\
+ \\
-\end{array}$ & $\begin{array}{l}- \\
- \\
- \\
-\end{array}$ & $\begin{array}{l}- \\
- \\
- \\
-\end{array}$ & $\begin{array}{l}- \\
- \\
- \\
-\end{array}$ & $\begin{array}{l}1: 3200 \\
1: 1600 \\
1: 800 \\
1: 1600 \\
1: 1600\end{array}$ \\
\hline $\begin{array}{l}11 \\
12 \\
13 \\
14 \\
15\end{array}$ & $\begin{array}{l}\text { K. M. } \\
\text { S. M. } \\
\text { O. M. } \\
\text { H. A. } \\
\text { Y. A. }\end{array}$ & $\begin{array}{l}46 \\
51 \\
31 \\
16 \\
29\end{array}$ & $\begin{array}{l}\mathrm{m} \\
\mathrm{m} \\
\mathrm{f} \\
\mathrm{f} \\
\mathrm{f}\end{array}$ & $\begin{array}{l}+ \\
+ \\
+ \\
+ \\
+\end{array}$ & $\begin{array}{l}+ \\
+ \\
+ \\
+ \\
+\end{array}$ & $\begin{array}{l}+ \\
+ \\
+ \\
+ \\
+\end{array}$ & $\begin{array}{l}- \\
- \\
+ \\
-\end{array}$ & $\begin{array}{l}- \\
- \\
- \\
-\end{array}$ & $\begin{array}{l}- \\
- \\
- \\
-\end{array}$ & $\begin{array}{l}1: 800 \\
1: 1600 \\
1: 800 \\
1: 1600 \\
1: 800\end{array}$ \\
\hline $\begin{array}{l}16 \\
17 \\
18 \\
19 \\
20\end{array}$ & $\begin{array}{l}\text { T. T. } \\
\text { M. A. } \\
\text { Y. A. } \\
\text { H. M. } \\
\text { N. S. }\end{array}$ & $\begin{array}{l}31 \\
34 \\
33 \\
32 \\
43\end{array}$ & $\begin{array}{l}f \\
f \\
f \\
f \\
f\end{array}$ & $\begin{array}{l}+ \\
+ \\
+ \\
+ \\
+\end{array}$ & $\begin{array}{l}+ \\
+ \\
+ \\
+ \\
+\end{array}$ & $\begin{array}{l}+ \\
+ \\
+ \\
+ \\
+\end{array}$ & $\begin{array}{l}- \\
- \\
+ \\
+ \\
-\end{array}$ & $\begin{array}{l}- \\
- \\
- \\
-\end{array}$ & $\begin{array}{l}- \\
- \\
- \\
- \\
-\end{array}$ & $\begin{array}{l}1: 1600 \\
1: 1600 \\
1: 1600 \\
1: 3200 \\
1: 800\end{array}$ \\
\hline $\begin{array}{l}21 \\
22 \\
23 \\
24 \\
25\end{array}$ & $\begin{array}{l}\text { T. M. } \\
\text { R. I. } \\
\text { T. N, } \\
\text { T. O. } \\
\text { K. O. }\end{array}$ & $\begin{array}{l}20 \\
54 \\
45 \\
39 \\
23\end{array}$ & $\begin{array}{l}\mathrm{f} \\
\mathrm{m} \\
\mathrm{m} \\
\mathrm{m} \\
\mathrm{m}\end{array}$ & $\begin{array}{l}+ \\
+ \\
+ \\
+ \\
+\end{array}$ & $\begin{array}{l}+ \\
+ \\
+ \\
+ \\
+\end{array}$ & $\begin{array}{l}- \\
+ \\
+ \\
+ \\
+\end{array}$ & $\begin{array}{l}- \\
- \\
- \\
-\end{array}$ & $\begin{array}{l}- \\
- \\
- \\
-\end{array}$ & $\begin{array}{l}- \\
- \\
- \\
-\end{array}$ & $\begin{array}{l}1: 1600 \\
1: 800 \\
1: 400 \\
1: 800 \\
1: 1600\end{array}$ \\
\hline $\begin{array}{l}26 \\
27 \\
28 \\
29 \\
30\end{array}$ & $\begin{array}{l}\text { Y. T. } \\
\text { S. E. } \\
\text { Y. T. } \\
\text { M. M. } \\
\text { H. N. }\end{array}$ & $\begin{array}{l}20 \\
32 \\
33 \\
31 \\
27\end{array}$ & $\begin{array}{l}\mathrm{m} \\
\mathrm{f} \\
\mathrm{m} \\
\mathrm{m} \\
\mathrm{m}\end{array}$ & $\begin{array}{l}+ \\
+ \\
+ \\
+ \\
+\end{array}$ & $\begin{array}{l}+ \\
+ \\
+ \\
+ \\
+\end{array}$ & $\begin{array}{l}+ \\
+ \\
+ \\
+ \\
-\end{array}$ & $\begin{array}{l}- \\
+ \\
- \\
-\end{array}$ & $\begin{array}{l}- \\
- \\
- \\
-\end{array}$ & $\begin{array}{l}- \\
- \\
- \\
-\end{array}$ & $\begin{array}{l}1: 800 \\
1: 800 \\
1: 1600 \\
1: 800 \\
1: 1600\end{array}$ \\
\hline $\begin{array}{l}31 \\
32 \\
33 \\
34 \\
3 \ddot{0}\end{array}$ & $\begin{array}{l}\text { E. M. } \\
\text { I. M. } \\
\text { T. M. } \\
\text { H. N. } \\
\text { K. I. }\end{array}$ & $\begin{array}{l}30 \\
35 \\
38 \\
53 \\
48\end{array}$ & $\begin{array}{l}\mathrm{m} \\
\mathrm{m} \\
\mathrm{f} \\
\mathrm{f} \\
\mathrm{f}\end{array}$ & $\begin{array}{l}+ \\
+ \\
+ \\
+ \\
+\end{array}$ & $\begin{array}{l}+ \\
+ \\
+ \\
+ \\
+\end{array}$ & $\begin{array}{l}+ \\
+ \\
+ \\
+ \\
+\end{array}$ & $\begin{array}{l}- \\
- \\
\overline{+} \\
+\end{array}$ & $\begin{array}{l}- \\
- \\
- \\
\overline{+}\end{array}$ & $\begin{array}{l}- \\
- \\
- \\
-\end{array}$ & $\begin{array}{l}1: 800 \\
1: 800 \\
1: 800 \\
1: 1600 \\
1: 1600\end{array}$ \\
\hline $\begin{array}{l}36 \\
37 \\
38 \\
39 \\
40\end{array}$ & $\begin{array}{ll}\text { M. } & \text { M. } \\
\text { S. } & \text { N. } \\
\text { M. } & \text { E. } \\
\text { M. } & \text { S. } \\
\text { M. N. N. }\end{array}$ & $\begin{array}{l}37 \\
42 \\
31 \\
33 \\
52\end{array}$ & $\begin{array}{l}f \\
f \\
f \\
f \\
f\end{array}$ & $\begin{array}{l}+ \\
+ \\
+ \\
+ \\
+\end{array}$ & $\begin{array}{l}+ \\
+ \\
+ \\
+ \\
+\end{array}$ & $\begin{array}{l}- \\
+ \\
+ \\
+ \\
+\end{array}$ & $\begin{array}{l}- \\
- \\
- \\
+\end{array}$ & $\begin{array}{l}- \\
\overline{-} \\
\overline{-}\end{array}$ & $\begin{array}{l}- \\
- \\
- \\
-\end{array}$ & $\begin{array}{l}1: 3200 \\
1: 1600 \\
1: 1600 \\
1: 1600 \\
1: 1600\end{array}$ \\
\hline $\begin{array}{l}41 \\
42 \\
43 \\
44 \\
45\end{array}$ & $\begin{array}{l}\text { K. Y. } \\
\text { S. O. } \\
\text { Y. O. } \\
\text { T. Y. } \\
\text { M. Y. }\end{array}$ & $\begin{array}{l}35 \\
24 \\
43 \\
35 \\
36\end{array}$ & $\begin{array}{l}f \\
f \\
f \\
f \\
m\end{array}$ & $\begin{array}{l}+ \\
+ \\
+ \\
+ \\
+\end{array}$ & $\begin{array}{l}+ \\
+ \\
+ \\
+ \\
+\end{array}$ & $\begin{array}{l}+ \\
+ \\
+ \\
+ \\
+\end{array}$ & $\begin{array}{l}+ \\
+ \\
+ \\
-\end{array}$ & $\begin{array}{l}- \\
- \\
- \\
-\end{array}$ & $\begin{array}{l}- \\
\overline{-} \\
- \\
-\end{array}$ & $\begin{array}{l}1: 1600 \\
1: 800 \\
1: 800 \\
1: 800 \\
1: 1600\end{array}$ \\
\hline
\end{tabular}




\begin{tabular}{|c|c|c|c|c|c|c|c|c|c|c|}
\hline No. & Name & Age & Sex & $1: 1$ & $1: 2$ & $1: 4$ & $1: 8$ & $1: 16$ & Cont. & $\begin{array}{l}\text { Serum precipitin } \\
\text { reaction }\end{array}$ \\
\hline 46 & Y. S. & 60 & $\mathrm{~m}$ & + & + & + & _- & - & - & $1: 1600$ \\
\hline 47 & I. Y. & 57 & $\mathrm{~m}$ & + & + & + & - & - & - & $1: 800$ \\
\hline 48 & Y. W. & 41 & $\mathrm{~m}$ & + & + & - & - & - & - & $1: 3200$ \\
\hline 49 & K. H. & 42 & $\mathrm{~m}$ & + & + & - & - & - & - & $1: 3200$ \\
\hline 50 & H. S. & 48 & $\mathbf{m}$ & + & + & + & - & - & - & $1: 1600$ \\
\hline 51 & S. Y. & 44 & $\mathrm{~m}$ & + & + & + & + & - & - & $1: 3200$ \\
\hline 52 & S. I. & 61 & $\mathbf{m}$ & + & + & + & - & - & - & $1: 800$ \\
\hline 53 & I. K. & 36 & $\mathbf{m}$ & + & + & + & - & - & - & $1: 3200$ \\
\hline 54 & K. N. & 17 & $\mathrm{~m}$ & + & + & + & + & - & - & \\
\hline 55 & T. K. & 20 & $\mathrm{~m}$ & + & + & - & - & - & - & $1: 3200$ \\
\hline 56 & K. H. & 37 & $\mathrm{f}$ & + & + & + & - & - & - & $1: 3200$ \\
\hline 57 & H. T. & 43 & $\mathrm{~m}$ & + & + & + & - & - & - & $1: 800$ \\
\hline 58 & Y. T. & 39 & $\mathrm{~m}$ & + & + & + & - & - & - & $1: 1600$ \\
\hline 59 & K. K. & 30 & $\mathbf{m}$ & + & + & + & + & + & - & $1: 800$ \\
\hline 60 & H. H. & 55 & $\mathrm{~m}$ & + & + & - & - & - & - & $1: 800$ \\
\hline 61 & H. H. & 27 & $\mathrm{~m}$ & + & + & + & - & - & - & $1: 800$ \\
\hline 62 & Y. M. & 28 & $\mathrm{~m}$ & + & + & + & - & - & - & $1: 800$ \\
\hline 63 & H. U. & 48 & $\mathrm{~m}$ & + & + & + & + & - & - & $1: 400$ \\
\hline 64 & T. O. & 12 & $\mathbf{m}$ & + & + & + & - & - & - & $1: 1600$ \\
\hline 65 & K. H. & 11 & $\mathrm{~m}$ & + & + & + & + & - & - & $1: 800$ \\
\hline 66 & H. K. & 12 & $\mathrm{~m}$ & + & + & + & - & - & - & $1: 1600$ \\
\hline 67 & T. K. & 12 & $\mathrm{~m}$ & t & + & + & - & - & - & $1: 1600$ \\
\hline 68 & H. M. & 11 & $\mathrm{~m}$ & + & + & - & - & - & - & $1: 800$ \\
\hline 69 & H. Y. & 10 & $\mathrm{f}$ & + & + & + & - & - & - & $1: 800$ \\
\hline 70 & E. H. & 12 & $\mathrm{f}$ & + & + & - & - & - & - & $1: 800$ \\
\hline 71 & I. I. & 10 & $f$ & + & + & + & - & - & - & $1: 1600$ \\
\hline 72 & H. I. & 12 & $\mathrm{f}$ & + & + & + & + & - & - & $1: 1600$ \\
\hline 73 & Y. O. & 10 & $\mathrm{f}$ & + & + & + & - & - & - & $1: 1600$ \\
\hline 74 & J. O. & 12 & $\mathrm{f}$ & + & + & - & - & - & - & $1: 800$ \\
\hline 75 & S. Y. & 12 & $\mathrm{f}$ & + & - & - & - & - & - & $1: 800$ \\
\hline 76 & Y. W. & 12 & $\mathrm{~m}$ & + & + & + & - & - & - & $1: 800$ \\
\hline 77 & T. K. & 11 & $\mathrm{~m}$ & + & + & - & - & 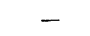 & - & $1: 1600$ \\
\hline 78 & $\mathrm{Y} . \mathrm{H}$ & 12 & $\mathrm{f}$ & + & + & - & - & - & - & $1: 1600$ \\
\hline 79 & K. H. & 12 & $\mathrm{f}$ & + & + & + & - & - & - & $1: 800$ \\
\hline 80 & N. O. & 9 & $\mathrm{~m}$ & + & + & + & - & - & - & $1: 1600$ \\
\hline 81 & J. O. & 12 & $\mathrm{~m}$ & + & + & + & - & - & - & $1: 3200$ \\
\hline 82 & T. K. & 12 & $\mathrm{~m}$ & + & + & + & + & - & - & $1: 1600$ \\
\hline 83 & S. T. & 11 & $\mathrm{~m}$ & + & - & - & - & - & - & $1: 200$ \\
\hline 84 & T. S. & 10 & $\mathrm{f}$ & + & + & + & - & - & - & $1: 800$ \\
\hline 85 & S. Y. & 11 & $\mathrm{~m}$ & + & + & - & - & - & - & $1: 1600$ \\
\hline 86 & T. H. & 11 & $\mathrm{~m}$ & + & + & + & - & - & - & $1: 800$ \\
\hline 87 & Y. K. & 9 & $\mathrm{~m}$ & + & + & - & - & - & - & $1: 1600$ \\
\hline 88 & M. N. & 7 & $\mathrm{~m}$ & + & + & + & - & - & - & \\
\hline 89 & T. S. & 8 & $\mathbf{m}$ & + & - & - & - & - & - & $1: 800$ \\
\hline 90 & H. Y. & 8 & $\mathrm{~m}$ & + & + & + & 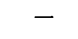 & & & \\
\hline & Y. N. & 9 & $\mathrm{~m}$ & + & + & + & - & - & - & \\
\hline 92 & M. O. & 9 & $\mathrm{~m}$ & + & + & + & - & - & - & \\
\hline 93 & S. Y. & 9 & $\mathrm{~m}$ & + & + & + & + & - & - & \\
\hline 94 & Y. K. & 9 & $\mathrm{~m}$ & + & + & + & - & - & - & \\
\hline 95 & H. S. & 10 & $\mathrm{~m}$ & + & + & - & - & - & - & $1: 800$ \\
\hline
\end{tabular}




\begin{tabular}{r|cc|c|c|c|c|c|c|c|c|c}
\hline 96 & Y. Y. & 10 & $\mathrm{~m}$ & + & + & + & + & - & - & \\
97 & K. Y. & 10 & $\mathrm{~m}$ & + & + & - & - & - & - & \\
98 & Y. & Y. & 11 & $\mathrm{~m}$ & + & + & - & - & - & - & $1: 800$ \\
99 & S. K. & 11 & $\mathrm{~m}$ & + & + & - & - & - & - & \\
100 & R. Y. & 11 & $\mathrm{f}$ & + & + & + & - & - & - & \\
101 & E. S. & 11 & $\mathrm{f}$ & + & + & - & - & - & - & & \\
102 & K. & Y. & 11 & $\mathrm{~m}$ & + & + & + & + & - & - & $1: 800$ \\
\hline
\end{tabular}

TABLE 4

The titer of urine and the distribution of the cases

\begin{tabular}{|c|c|c|}
\hline Titer of Urine & Cases & $\%$ \\
\hline $1: 1$ & 3 & 2.9 \\
\hline $1: 2$ & 23 & 22.6 \\
\hline $1: 4$ & 55 & 53.9 \\
\hline $1: 8$ & 18 & 17.6 \\
\hline $1: 16$ & 3 & 2.9 \\
\hline Total & 102 & \\
\hline
\end{tabular}

More than the half of the examined showed a titer of $1: 4$.

4. Normal persons

The urine precipitin reactions of ten normal persons were examined with Schistosoma immune serum, but all were negative.

\section{Group reactions}

a. The urine of Schistosoma mansoni infected rabbits.

Two rabbits were infected with 50 cercariae of Schistosoma mansoni got from Australorbis glabratus. The difference of the urine precipitin reaction was examined before and after the infection. Before the infection the reaction of the two rabbits was negative, and in 59 and 63 days after the infection it turned out to be positive respectively, but their titers were very low.

TABLE 5

The urine precipitin reaction between the urine obtained from Schistosoma mansoni infected rabbits and Schistosoma japonicum immunized rabbit serum

\begin{tabular}{c|c|c|c|c|c|c|c}
\hline No. & Days after infection & $1: 1$ & $1: 2$ & $1: 4$ & $1: 8$ & $1: 16$ & Cont. \\
\hline \multirow{6}{*}{1} & Before infection & - & - & - & - & - & - \\
& 7 days & - & - & - & - & - & - \\
& 21 days & - & - & - & - & - & - \\
& 35 days & - & - & - & - & - & - \\
& 49 days & - & - & - & - & - & - \\
& 63 days & + & - & - & - & - & - \\
\hline \multirow{6}{*}{77 days } & \pm & - & - & - & - & - \\
& Before infection & - & - & - & - & - & - \\
2 & 3 days & - & - & - & - & - & - \\
& 17 days & - & - & - & - & - & - \\
& 31 days & - & - & - & - & - & - \\
& 45 days & - & - & - & - & - & - \\
& 59 days & + & \pm & - & - & - & - \\
\hline
\end{tabular}


b. Paragonimiasis patients

Four cases out of 11 paragonimiasis patients were positive at a titer of $1: 1$. Group reaction was observed, but their titer was low.

c. Clonorchiasis patients

The urine precipitine reactions of five cases of clonorchiasis were examined, but all were negative.

\section{NATURE OF THE URINE ANTIGEN}

\section{The influence of temperature at the urine concentration}

The urine titer was examined at various temperatures during the concentration. The reaction did not appear when the temperature was $80^{\circ} \mathrm{C}$ and higher.

$$
\text { TABLE } 6
$$

The influence of temperature on the antigen at the urine concentration

\begin{tabular}{|c|c|c|c|c|c|c|c|c|c|c|c|c|c|c|c|c|}
\hline \multirow{2}{*}{ Tem perature } & \multirow{2}{*}{ Minutes } & \multicolumn{5}{|c|}{ No. 1} & \multicolumn{5}{|c|}{ No. 2} & \multicolumn{5}{|c|}{ No. 3} \\
\hline & & $1: 1$ & $1: 2$ & $1: 4$ & $1: 8$ & Cont. & $1: 1$ & $1: 2$ & $1: 4$ & $1: 8$ & Cont. & $1: 1$ & $1: 2$ & $1: 4$ & $1: 8$ & Cont. \\
\hline $40^{\circ} \mathrm{C}$ & $30 \mathrm{~min}$ & + & + & + & - & - & + & + & + & - & - & + & + & + & - & - \\
\hline $50^{\circ} \mathrm{C}$ & $25 \mathrm{~min}$ & + & + & + & - & - & + & + & + & - & - & + & + & + & - & - \\
\hline $60^{\circ} \mathrm{C}$ & $15 \mathrm{~min}$ & + & + & + & - & - & + & + & - & - & - & + & + & + & - & - \\
\hline $70^{\circ} \mathrm{C}$ & $10 \mathrm{~min}$ & + & + & - & - & - & + & + & - & - & - & + & - & - & - & - \\
\hline $80^{\circ} \mathrm{C}$ & $7 \mathrm{~min}$ & - & - & - & - & - & - & - & - & - & - & - & - & - & - & - \\
\hline $90^{\circ} \mathrm{C}$ & $3 \min$. & - & - & - & - & - & - & - & - & - & - & - & - & - & - & - \\
\hline $100^{\circ} \mathrm{C}$ & $3 \mathrm{~min}$. & - & - & - & - & - & - & - & - & - & - & - & - & - & - & - \\
\hline
\end{tabular}

\section{Preservation of the urine antigen}

The urine was putrescible in summer. Sometimes the next day the urine was found decomposed. Even if it was stored in a refrigerator, it could be used for only two days.

When the sodium azide was added to it at a dilution of $0.1 \%$, at the room temperature $12-19^{\circ} \mathrm{C}$, the titer of urine had not changed for six days after the urination, but on the 9 th day the titer of urine dropped and on the 12th day one of three samples was negative.

TABLE 7

Preservation of urine at $12^{\circ}-19^{\circ} \mathrm{C}$

a) The urine containing $0.1 \% \mathrm{NaN}_{3}$.

\begin{tabular}{|c|c|c|c|c|c|c|c|c|c|c|c|c|c|c|c|}
\hline \multirow{3}{*}{$\begin{array}{c}\text { Days after urination } \\
0\end{array}$} & \multicolumn{5}{|c|}{ No. 1} & \multicolumn{5}{|c|}{ No. 2} & \multicolumn{5}{|c|}{ No. 3} \\
\hline & \multicolumn{2}{|c|}{$1: 1 \mid 1: 2$} & \multicolumn{2}{|c|}{$1: 4: 8$} & \multirow{2}{*}{$\frac{\mid \text { Cont. }}{-}$} & \multicolumn{2}{|c|}{$1: 11: 2$} & \multicolumn{2}{|c|}{$1: 41: 8$} & \multirow{2}{*}{$\frac{\mid \text { Cont. }}{-}$} & \multicolumn{2}{|c|}{$1: 1 \mid 1: 2$} & \multicolumn{2}{|c|}{$1: 41: 8$} & \multirow{2}{*}{ Cont. } \\
\hline & + & + & + & - & & + & + & + & - & & + & + & + & - & \\
\hline 3 & + & + & + & - & - & + & + & + & - & - & + & + & + & - & - \\
\hline 6 & + & + & - & - & - & + & + & + & - & - & + & + & + & - & - \\
\hline 9 & + & + & - & - & - & + & + & - & - & - & + & + & - & - & - \\
\hline 12 & - & - & - & - & - & + & - & - & - & - & + & - & - & - & - \\
\hline
\end{tabular}


b) The urine without $\mathrm{NaN}_{3}$.

\begin{tabular}{|c|c|c|c|c|c|c|c|c|c|c|c|c|c|c|c|}
\hline \multirow{2}{*}{ Days after urination } & \multicolumn{5}{|c|}{ No. 1} & \multicolumn{5}{|c|}{ No. 2} & \multicolumn{5}{|c|}{ No. 3} \\
\hline & \multicolumn{4}{|c|}{$1: 11: 21: 41: 8$} & \multirow{2}{*}{\begin{tabular}{|c|} 
Cont. \\
-
\end{tabular}} & \multicolumn{2}{|c|}{$1: 11: 2$} & \multicolumn{2}{|c|}{$1: 41: 8$} & \multirow{2}{*}{$\frac{3 \text { Cont. }}{-}$} & \multicolumn{2}{|c|}{$1: 11: 2$} & $1: 4$ & $1: 8$ & Cont. \\
\hline 0 & + & + & + & - & & + & + & + & - & & + & + & + & - & - \\
\hline 3 & \multicolumn{5}{|c|}{ opacitas } & + & + & + & - & - & + & + & + & - & - \\
\hline 6 & \multicolumn{5}{|c|}{ opacitas } & \multicolumn{5}{|c|}{ opacitas } & \multicolumn{5}{|c|}{ opacitas } \\
\hline
\end{tabular}

\section{The cultured medium}

Four pairs of Schistosoma japonicum were removed from a rabbit and were cultured, two with saline solution and the other two with Ringer's solution in Carrel flasks. The cultured medium was used as an antigen for the precipitin test. The titer of the medium was $1: 1$ after 5 hours cultivation and $1: 2$ after 10 hours cultivation in $0.85 \% \mathrm{NaCl}$ solution. It was $1: 2$ after 12 hours cultivation and the same after 24 hours in Ringer's solution containing $0.5 \%$ glucose.

TABLE 8

The precipitin reaction between the cultured medium of Schistosoma japonicum

and Schistosoma japonicum immunized rabbit serum

a) $0.85 \% \mathrm{NaCl}$ solution

\begin{tabular}{r|c|c|c|c|c|c}
\hline Hours & $1: 1$ & $1: 2$ & $1: 4$ & $1: 8$ & $1: 16$ & Cont. \\
\hline 5 hours & + & - & - & - & - & - \\
10 hours & + & + & - & - & - & - \\
\hline
\end{tabular}

b) Ringer's solution containing $0.5 \%$ glucose

\begin{tabular}{c|c|c|c|c|c|c}
\hline Hours & $1: 1$ & $1: 2$ & $1: 4$ & $1: 8$ & $1: 16$ & Cont. \\
\hline 12 hours & + & + & - & - & - & - \\
24 hours & + & + & - & - & - & - \\
\hline
\end{tabular}

\section{ASSESSMENT OF CURE}

\section{TWSb treatment}

Five patients of schistosomiasis japonica were injected intramuscularly with antimony-a, a'-potassium succinate (TWSb).

Three male patients were injected with $0.3-0.4 \mathrm{~g}$ TWSb totaling 2.05-2.28g, and two female patients were injected $0.3-0.4 \mathrm{~g}$ TWSb totaling $1.5-1.7 \mathrm{~g}$ during a week. The therapeutic effects had been examined observing the eggs in feces, the titer of urine and serum precip tin reactions of the patients during the course of about one year after the treatment.

The fecal examination, after three days of the treatment, revealed that three of them were negative, but two patients were still positive. After 77 days no eggs were found in the feces of any of them and by the examination after about one year they were still negative.

The urine precipitin reaction was positive in all cases for three days after the treatment. After 43 days the reaction of one of them turned negative, and that of the four were positive at a titer of $1: 1$. After 78 days the reaction of all proved negative and remained negative after one year. It could be considered to be certain that no recidivation and no reinfection occurred during the year. (It must be added here the fact that there was not found any Oncomelania 
snail in the field where these patients were working during the year.)

The serum precipitin reactions were positive with all cases on the $43 \mathrm{th}, 77 \mathrm{th}$ and 69th day after the treatment, while the titer lended to drop. One year after the treatment the reaction of four patients was negative and the titer of the one positive cases was at a dilution of $1: 100$.

TABLE 9

Course of the urine precipitin reactions and fecal examinations after treatment with $T W S b$

\begin{tabular}{|c|c|c|c|c|c|c|c|c|c|c|c|c|}
\hline Name & $\begin{array}{r}\mathrm{Da} \\
\text { examir }\end{array}$ & $\begin{array}{l}\text { ate } \\
\text { ination }\end{array}$ & $\begin{array}{l}\text { Days after } \\
\text { treatment }\end{array}$ & $\mid \begin{array}{c}\text { Fecal } \\
\text { examination }\end{array}$ & $1: 1$ & $1: 2$ & $1: 4$ & $1: 8$ & $1: 16$ & $1: 32$ & Cont. & $\begin{array}{l}l \text { lmmune } \\
\text { Serum } \\
\text { t:ter }\end{array}$ \\
\hline \multirow{9}{*}{ M. I. } & Feb. & 8 & $\begin{array}{l}\text { Before } \\
\text { treatment }\end{array}$ & + & t & + & + & - & - & - & - & $1: 3200$ \\
\hline & Feb. 1 & & 4 days & - & + & + & + & - & - & - & - & " \\
\hline & Marrh : & & 44 days & - & + & - & - & - & - & - & - & $"$ \\
\hline & May & 3 & 78 days & - & - & - & - & - & - & - & - & " \\
\hline & June & 10 & 115 days & & - & - & - & - & - & - & - & " \\
\hline & Aug. & 4 & 170 days & & - & - & - & - & - & - & - & $1: 6400$ \\
\hline & Sept. & 16 & 213 days & - & - & - & - & - & - & - & - & $1: 3200$ \\
\hline & Oct. & 7 & 237 days & - & - & - & - & - & - & - & - & $"$ \\
\hline & Jan. & 28,1959 & 348 days & - & - & - & - & - & - & - & - & " \\
\hline \multirow{9}{*}{ M. M. } & Feb. & 8 & $\begin{array}{l}\text { Before } \\
\text { treatment }\end{array}$ & + & + & + & + & + & - & - & - & $1: 3200$ \\
\hline & Feb. 1 & 18 & 4 days & + & + & + & + & - & - & - & - & $"$ \\
\hline & March 3 & & 44 days & - & - & - & - & - & - & - & - & " \\
\hline & May & 3 & 78 days & - & - & - & - & - & - & - & - & " \\
\hline & June & 10 & 115 days & & - & - & - & - & - & - & - & " \\
\hline & Aug. & 4 & 170 days & & \pm & - & - & - & - & - & - & $1: 6400$ \\
\hline & Sept. & 16 & 213 days & - & $=$ & - & - & - & - & - & - & $1: 3200$ \\
\hline & Oct. & 7 & 237 days & - & - & - & - & - & - & - & - & " \\
\hline & Jan. & 28,1959 & 348 days & - & - & - & - & - & - & - & - & " \\
\hline \multirow{9}{*}{ T. E. } & Feb. & 8 & $\begin{array}{l}\text { Before } \\
\text { treatment }\end{array}$ & + & + & + & + & + & + & - & - & $1: 3200$ \\
\hline & Feb. $\quad 1$ & 18 & 4 days & - & + & + & + & + & - & - & - & " \\
\hline & March & 30 & $44 \mathrm{~d}$ & - & + & \pm & $r$ & - & 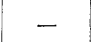 & - & - & " \\
\hline & May & 3 & 78 days & - & - & - & - & - & - & - & - & " \\
\hline & June & 10 & 115 days & & - & - & - & - & - & - & - & " \\
\hline & Aug. & 4 & 170 days & & - & - & - & - & - & - & - & $1: 6400$ \\
\hline & Sept. & 16 & 213 days & - & - & - & - & - & - & - & - & $1: 3200$ \\
\hline & Oct. & 7 & 237 days & - & - & - & - & - & - & - & - & " \\
\hline & Jan. & 28,1959 & 348 days & - & - & - & - & - & - & 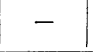 & - & $"$ \\
\hline \multirow{9}{*}{ Y. I. } & Feb. & 8 & $\begin{array}{l}\text { Before } \\
\text { treatment }\end{array}$ & + & + & + & + & - & - & - & - & $1: 3200$ \\
\hline & Feb. $\quad 1$ & 18 & 4 days & - & + & + & + & - & - & - & - & " \\
\hline & March & 30 & 44 days & - & + & - & . & - & - & - & - & " \\
\hline & May & 3 & 78 days & - & - & - & -- & - & - & - & - & " \\
\hline & June & 10 & 115 days & & - & - & - & - & - & - & - & " \\
\hline & Aug. & 4 & 170 days & & - & - & - & - & - & - & - & $1: 6400$ \\
\hline & Sept. & 16 & 213 days & - & - & - & - & - & - & - & - & $1: 3200$ \\
\hline & Oct. & 7 & 237 days & - & - & - & - & - & - & - & - & " \\
\hline & Jan. & 28,1959 & 348 days & - & - & - & - & - & - & . & - & $"$ \\
\hline
\end{tabular}




\begin{tabular}{|c|c|c|c|c|c|c|c|c|c|c|c|c|}
\hline \multirow{9}{*}{ T. I. } & Feb. & 8 & $\begin{array}{l}\text { Before } \\
\text { treatmen }\end{array}$ & + & + & + & + & + & - & - & - & $1: 3200$ \\
\hline & Feb. & 18 & 4 days & - & + & + & \pm & - & - & - & - & $"$ \\
\hline & March & 30 & 44 dayt & - & + & - & - & - & - & - & - & " \\
\hline & May & 3 & 78 days & - & - & - & - & - & - & - & - & " \\
\hline & June & 10 & 115 days & & - & - & - & - & - & - & - & " \\
\hline & A ug. & 4 & 170 days & & - & - & - & - & - & - & - & $1: 6400$ \\
\hline & Sept. & 16 & 213 days & - & - & - & - & - & - & - & - & $1: 3200$ \\
\hline & Oct. & 7 & 237 daxs & - & - & - & - & - & - & - & - & " \\
\hline & Jan. & 28,1959 & 348 days & - & - & - & - & - & - & - & - & " \\
\hline
\end{tabular}

TABLE 10

Course of the serum precipitin reactions and fecal examinations after treatment

\begin{tabular}{|c|c|c|c|c|c|c|c|c|c|c|c|c|}
\hline Name & $\begin{array}{l}\text { Date } \\
\text { exam }\end{array}$ & ined & $\begin{array}{l}\text { Days after } \\
\text { treatment }\end{array}$ & $\begin{array}{l}\text { Fecal ex- } \\
\text { amination }\end{array}$ & $1:$ & 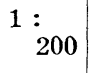 & $1:$ & $1:$ & $1:$ & $\begin{array}{l}1: \\
3200\end{array}$ & 1: & Cont. \\
\hline \multirow{5}{*}{ M. I. } & & & $\begin{array}{l}\text { Before } \\
\text { treatment }\end{array}$ & + & + & + & + & + & + & \pm & - & - \\
\hline & March & & 43 days & - & + & + & + & + & + & \pm & - & - \\
\hline & May & 2 & 77 days & - & + & + & + & - & - & - & - & - \\
\hline & Aug. & 3 & 169 days & - & + & + & + & - & - & - & - & - \\
\hline & Jan. & 27,1959 & 347 days & - & - & - & - & - & - & - & - & - \\
\hline \multirow{5}{*}{ M. M. } & & & $\begin{array}{l}\text { Before } \\
\text { treatment }\end{array}$ & + & + & + & + & + & \pm & - & - & - \\
\hline & March & 29 & 43 pays & + & + & + & + & + & + & \pm & - & - \\
\hline & May & 2 & 77 days & - & + & + & + & - & - & - & - & - \\
\hline & Aug. & 3 & 169 days & - & + & + & - & - & - & - & - & - \\
\hline & Jan. & 27,1959 & 347 days & - & + & - & - & - & - & - & - & - \\
\hline \multirow{5}{*}{ T. E. } & Feb. & & $\begin{array}{l}\text { Before } \\
\text { treatment }\end{array}$ & + & + & + & + & + & + & \pm & - & - \\
\hline & March & 29 & 43 days & + & + & + & + & + & + & - & - & - \\
\hline & May & 2 & 77 days & - & + & + & + & \pm & - & - & - & - \\
\hline & Aug. & 3 & 169 days & - & + & + & + & - & - & - & - & - \\
\hline & Jan. & 27,1959 & 347 days & - & - & - & - & - & - & - & - & - \\
\hline \multirow{5}{*}{ Y. I. } & Feb. & & $\begin{array}{l}\text { Before } \\
\text { treatment }\end{array}$ & + & + & + & + & + & + & - & - & - \\
\hline & March & 29 & 43 days & - & + & + & + & + & + & - & - & - \\
\hline & May & 2 & 77 days & - & + & + & + & - & - & - & - & - \\
\hline & Aug. & 3 & 169 days & - & + & + & + & - & - & - & - & - \\
\hline & Jan. & 27,1959 & 347 days & - & - & - & - & - & - & - & - & - \\
\hline \multirow{5}{*}{ T. I. } & Feb. & 8 & $\begin{array}{l}\text { Before } \\
\text { treatment }\end{array}$ & + & + & + & + & + & - & - & - & - \\
\hline & March & 29 & 43 days & - & + & + & + & + & \pm & - & - & - \\
\hline & May & 2 & 77 days & - & + & + & + & - & - & - & - & - \\
\hline & Aug. & 3 & 169 days & - & + & + & - & - & - & - & - & - \\
\hline & Jan. & 27,1959 & 347 days & - & - & - & - & $T$ & - & - & - & - \\
\hline
\end{tabular}




\section{Stibnal (Sodium antimony tartrate)}

Twenty chronic patients were treated with Stibnal $(0.3 \%$ sodium antimony tartrate) and followed up with the urine precipitin reaction. $20 \mathrm{ml}$ Stibnal was injected to each patient 13-50 times respectively. In Japan Stibnal is injected 20 times usually. By our experiment, about the cases with 27-50 times injection the reaction was negative, but about the cases injected 26-13 times the reaction was positive with one exception. These results imply that 20 times injection of Stibnal was not sufficient to be curative.

TABLE 11

The urine precipitin reaction of treated cases with Stibnal

\begin{tabular}{|c|c|c|c|c|c|c|c|c|c|c|c|}
\hline Name & Age & Sex & $\begin{array}{l}\text { Times of } \\
\text { in jection }\end{array}$ & $\begin{array}{l}\text { Months after } \\
\text { treatment }\end{array}$ & $1: 1$ & $1: 2$ & $1: 4$ & $1: 8$ & $1: 16$ & $1: 32$ & Cont. \\
\hline T. O. & 44 & $\mathrm{~m}$ & 50 & 2 & - & - & - & - & - & - & - \\
\hline T. I. & 42 & $\mathrm{~m}$ & 42 & 3 & - & - & - & - & - & - & - \\
\hline M. N. & 26 & $\mathrm{~m}$ & 40 & 13 & - & - & - & - & - & - & - \\
\hline M. M. & 33 & $\mathrm{f}$ & 36 & 28 & - & - & - & - & - & - & - \\
\hline Y. S. & 34 & $\mathrm{~m}$ & 33 & 8 & - & - & - & - & - & - & - \\
\hline S. T. & 44 & $f$ & 30 & 5 & - & - & - & - & - & - & - \\
\hline T. O. & 24 & $\mathrm{~m}$ & 30 & 3 & - & - & - & - & - & - & - \\
\hline S. T. & 34 & $\mathrm{~m}$ & 30 & 8 & - & - & - & - & - & - & - \\
\hline Y. N. & 28 & $\mathrm{~m}$ & 30 & 13 & - & - & - & - & - & - & - \\
\hline A. Y. & 50 & $\mathrm{f}$ & 27 & 58 & - & - & - & - & - & - & - \\
\hline S. A. & 38 & $f$ & 26 & 10 & + & + & - & - & - & - & - \\
\hline T. O. & 31 & $\mathrm{~m}$ & 25 & 10 & - & - & - & - & - & - & - \\
\hline H. M. & 14 & $\mathrm{~m}$ & 25 & 3 & + & - & - & - & - & - & - \\
\hline S. T. & 38 & $f$ & 25 & 8 & + & + & - & - & - & - & - \\
\hline Y. M. & 14 & $\mathrm{~m}$ & 20 & 9 & + & + & + & - & - & - & - \\
\hline Y. T. & 34 & $\mathrm{~m}$ & 20 & 57 & - & - & - & - & - & - & - \\
\hline K. K. & 67 & $\mathrm{~m}$ & 20 & 2 & + & + & - & - & - & - & - \\
\hline K. K. & 48 & $\mathrm{~m}$ & 20 & 8 & + & + & - & - & - & - & - \\
\hline K. T. & 13 & $\mathrm{~m}$ & 15 & 9 & + & + & + & - & - & - & - \\
\hline H. H. & 29 & f & 13 & 3 & + & + & + & - & - & - & - \\
\hline
\end{tabular}

3. Animal experiments

a. TWSb treatment

i. rabbits

TWSb was injected intramuscularly, $8 \mathrm{mg} / \mathrm{kg}$ a day, to Schistosoma infected rabbits for five days and its effects observed for 61 days. The urine

TABLE 12

The urine precipitin reaction of treated rabbits with $T W S b(40 \mathrm{mg} / \mathrm{kg}$ )

\begin{tabular}{c|c|c|c|c|c|c|c|c|c|c|c|c|c|c|c}
\hline $\begin{array}{c}\text { Days after } \\
\text { treatment }\end{array}$ & No. & $1: 1$ & $1: 2$ & $1: 4$ & $1: 8$ & $1: 16$ & Cont. & No. & $1: 1$ & $1: 2$ & $1: 4$ & $1: 8$ & $1: 16$ & Cont. \\
Before treat. & & + & + & - & - & - & - & & + & + & + & - & - & - \\
16 days & & + & + & - & - & - & - & & + & + & + & - & - & - \\
28 days & $\mathbf{9}$ & + & + & - & - & - & - & 10 & + & + & - & - & - & - \\
46 days & & + & + & + & - & - & - & & + & + & - & - & - & - \\
61 days & & + & + & - & - & - & - & & + & + & - & - & - & - \\
\hline
\end{tabular}


precipitin reaction did not turn out to be negative and some worms were found by autopsy.

ii. dogs

TWSb was injected intramuscularly, $8 \mathrm{mg} / \mathrm{kg}$ to the dogs infected with 150 cercariae of Schistosoma japonicum. After 105 days from the treatment the reaction changed to negative while the control dogs showed positive reactions. By autopsy there no Schistosoma was found in the treated dogs, while in the control dogs 29 and 55 worms were found respectively.

TABLE 13

Follow-up with fecal examination and urine precipitin reaction after the treatment of an infected dog with $T W S b(40 \mathrm{mg} / \mathrm{kg}$ in 5 days)

\begin{tabular}{|c|c|c|c|c|c|c|c|c|c|c|c|c|c|c|c|c|}
\hline \multirow{2}{*}{$\begin{array}{l}\text { Days } \\
\text { after } \\
\text { infection }\end{array}$} & \multirow{2}{*}{$\begin{array}{l}\text { Days } \\
\text { after } \\
\text { treatment }\end{array}$} & \multicolumn{5}{|c|}{ Treated dog } & \multicolumn{5}{|c|}{ Control dog No. 1} & \multicolumn{5}{|c|}{ Control dog No. 2} \\
\hline & & \multicolumn{4}{|c|}{$1: 11: 21: 41: 8$} & \multirow{2}{*}{$\frac{\text { eggs }}{+}$} & \multicolumn{4}{|c|}{$1: 11: 21: 41: 8$} & \multirow{2}{*}{$\frac{\text { eggs }}{+}$} & \multicolumn{4}{|c|}{$1: 11: 21: 41: 8$} & \multirow{2}{*}{$\begin{array}{c}\text { eggs } \\
+\end{array}$} \\
\hline 41 days & Before & + & + & + & - & & + & + & + & - & & + & + & + & - & \\
\hline 49 & 7 days & + & + & + & - & + & + & + & + & - & + & + & + & + & - & + \\
\hline 70 & 28 & + & + & - & - & - & + & + & + & - & + & + & + & - & - & + \\
\hline 77 & 35 & + & + & - & - & - & + & + & + & + & + & + & + & - & - & + \\
\hline 91 & 49 & + & - & - & - & - & + & + & + & - & + & + & + & + & - & + \\
\hline 147 & 105 & i & - & - & - & - & + & + & + & - & + & + & + & - & - & + \\
\hline 154 & 112 & $\mathrm{Au}$ & psy & C- & & & mal & 1 & eme & 15 & & & 20 & fem: & e 27 & \\
\hline
\end{tabular}

*Deformed egg

b. Stibnal treatment

Stibnal was injected intravenously, $0.5 \mathrm{ml} / \mathrm{kg}, 0.8 \mathrm{ml} / \mathrm{kg}$, and $1.0 \mathrm{ml} / \mathrm{kg}$ a day, to Schistosoma infected rabbits for twenty days and every other week after the treatment the urine precipitin reaction was tried on them and examined.

i. Stibnal $1.0 \mathrm{ml} / \mathrm{kg}$ for 20 days

No. 1 became negative on the 4 th week and No. 2 on the 5 th week. The next week the rabbits were dissected and no worms were found.

ii. Stibnal $0.8 \mathrm{ml} / \mathrm{kg}$ for 20 days

No. 3 did not change to be negative and was found to be still infected with worms ( 19 and 919 ). No. 4 became negative on the 4 th week and on the 5 th week, when it was dessected, two degenerated male worms were found in its liver by perfusion.

iii. Stibnal $0.5 \mathrm{ml} / \mathrm{kg}$ for 20 days

Both No. 5 and No. 6 did not become negative and were found to be still infected with worms. 
TABLE 14

The urine precipitin reaction of the treated rabbits with Stibnal a) Stibnal $1.0 \mathrm{ml} / \mathrm{kg}$ for 20 days

\begin{tabular}{|c|c|c|c|c|c|c|c|c|c|c|c|c|c|c|}
\hline $\begin{array}{l}\text { Days after } \\
\text { treatment }\end{array}$ & No. & $1: 1$ & $1: 2$ & $1: 4$ & $1: 8$ & $1: 16$ & Cont. & No. & $1: 1$ & $1: 2$ & $1: 4$ & $1: 8$ & $1: 16$ & Cont. \\
\hline Before treat. & & + & + & + & - & - & - & & + & + & + & + & - & - \\
\hline 1 week & & + & + & + & - & - & - & & + & + & + & - & - & - \\
\hline 2 weeks & 1 & + & + & - & - & - & - & ? & + & + & + & - & - & - \\
\hline 3 weeks & 1 & + & - & - & - & - & - & 2 & + & + & - & - & - & - \\
\hline 4 weeks & & - & - & - & - & - & - & & + & - & - & - & - & - \\
\hline 5 weeks & & - & - & - & - & - & - & & - & - & - & - & - & - \\
\hline
\end{tabular}

\begin{tabular}{c|c|c|c|c|c|c|c|c|c|c|c|c|c|c|c}
\hline \multicolumn{10}{c}{ b) } & \multicolumn{10}{l|l}{ Stibnal $0.8 \mathrm{ml} / \mathrm{kg}$ for 20 days } \\
\hline $\begin{array}{c}\text { Days after } \\
\text { treatment }\end{array}$ & No. & $1: 1$ & $1: 2$ & $1: 4$ & $1: 8$ & $1: 16$ & Cont. & No. & $1: 1$ & $1: 2$ & $1: 4$ & $1: 8$ & $1: 16$ & Cont. \\
\hline Before treat. & & + & + & + & - & - & - & & + & + & - & - & - & - \\
1 week & & + & + & + & + & - & - & & + & + & + & - & - & - \\
2 weeks & 3 & + & + & - & - & - & - & & + & + & - & - & - & - \\
3 weeks & & + & + & - & - & - & - & 4 & + & + & - & - & - & - \\
4 weeks & & + & + & - & - & - & - & & - & - & - & - & - & - \\
5 weeks & & + & + & - & - & - & - & & - & - & - & - & - & - \\
\hline
\end{tabular}

c) Stibnal $0.5 \mathrm{ml} / \mathrm{kg}$ for 20 days

\begin{tabular}{c|c|c|c|c|c|c|c|c||c|c|c|c|c|c|c|c}
\hline $\begin{array}{c}\text { Days after } \\
\text { treatment }\end{array}$ & No. $1: 1$ & $1: 2$ & $1: 4$ & $1: 8$ & $1: 16$ & Cont. & No. & $1: 1$ & $1: 2$ & $1: 4$ & $1: 8$ & $1: 16$ & Cont. \\
\hline Before treat. & & + & + & + & - & - & - & & + & + & + & + & - & - \\
1 week & & + & + & - & - & - & - & & + & + & - & - & - & - \\
2 weeks & 5 & + & + & + & - & - & - & & + & + & - & - & - & - \\
3 weeks & 5 & + & + & - & - & - & - & 6 & + & + & - & - & - & - \\
4 weeks & & + & + & - & - & - & - & & + & + & - & - & - & - \\
5 wəeks & & + & + & - & - & - & - & & + & + & + & - & - & - \\
\hline
\end{tabular}

\section{EARLY DIAGNOSIS}

It was showed in the previous part (III-1) that the acute cases of schistosomiasis japonica reacted to the urine precipitin reaction. Animal experiments TABLE 15

The urine precipitin reaction of infected rabhits

\begin{tabular}{|c|c|c|c|c|c|c|c|c|c|c|c|c|c|c|}
\hline No. & $\begin{array}{c}\begin{array}{c}\text { Days after } \\
\text { infection }\end{array} \\
\end{array}$ & $1: 1$ & $1: 2$ & $1: 4$ & $1: 8$ & $1: 16$ & Cont. & No. & $1: 1$ & $1: 2$ & $1: 4$ & $1: 8$ & $1: 16$ & Cont. \\
\hline \multirow{8}{*}{1} & Before inf. & - & - & - & - & - & - & \multirow{8}{*}{4} & - & - & - & - & - & - \\
\hline & 3 days & \pm & - & - & - & - & - & & + & - & - & - & - & - \\
\hline & 6 days & + & + & - & - & - & - & & + & + & - & - & - & 一 \\
\hline & 9 days & + & + & - & - & - & - & & + & + & - & - & - & - \\
\hline & 12 days & + & + & - & - & - & - & & + & + & - & - & - & - \\
\hline & 15 days & + & + & + & - & - & - & & + & + & + & - & - & - \\
\hline & 18 days & + & + & + & - & - & - & & + & + & - & - & - & - \\
\hline & 21 days & + & + & - & - & - & - & & + & + & + & - & - & - \\
\hline
\end{tabular}




\begin{tabular}{|c|c|c|c|c|c|c|c|c|c|c|c|c|c|c|}
\hline \multirow{8}{*}{2} & Before inf. & - & - & - & - & - & - & \multirow{8}{*}{5} & - & - & - & - & - & - \\
\hline & 3 days & + & - & - & - & - & - & & - & - & - & - & - & - \\
\hline & 6 days & + & + & - & - & - & - & & + & - & - & - & - & - \\
\hline & 9 days & + & + & + & - & - & - & & + & + & + & - & - & - \\
\hline & 12 days & + & + & - & - & - & - & & + & + & - & - & - & - \\
\hline & 15 days & + & + & t & - & - & - & & + & + & + & + & - & - \\
\hline & 18 days & + & + & + & - & - & - & & + & + & - & - & - & - \\
\hline & 21 days & + & + & + & - & - & - & & + & + & + & + & - & - \\
\hline \multirow{8}{*}{3} & Before inf. & - & - & - & - & - & - & \multirow{8}{*}{6} & - & - & - & - & - & - \\
\hline & 3 days & \pm & - & - & - & - & - & & \pm & - & - & - & - & - \\
\hline & 6 days & + & - & - & - & - & - & & t & - & - & - & - & - \\
\hline & 9 days & + & + & + & - & - & - & & + & t & - & - & - & - \\
\hline & 12 days & + & + & + & - & - & - & & t & + & + & - & - & - \\
\hline & 15 days & + & + & - & - & - & - & & + & + & + & - & - & - \\
\hline & 18 days & + & + & + & + & - & - & & + & + & + & - & - & - \\
\hline & 21 days & t & + & + & - & - & - & & + & + & - & - & - & - \\
\hline
\end{tabular}

will lead us to presume when the reaction turns to be positvie after the infection.

Two out of six rabbits, which had been infected with about one hundred cercariae, showed a titer of $1: 2$ and the others a titer of $1: 1$ on the 6th day. On the 9th day three of them became positive at a titer of $1: 4$ and others $1: 2$.

\section{SUMMARY}

The acute, sub-acute and chronic patients of schistosomiasis japonica reacted to the urine precipitin reaction. The antigen in urine is easy to change by heat and putrefaction. It is not clear what substance participates in this phenomenon, but it is presumable that the substance may be thermolabil and nondialyzable.

The urine precipitin reaction may be used for the assessment of cure. Animal experiments showed the fact that after the reaction had become negative, the worm was not found any more.

This reaction may be used for early diagnosis for schistosomiasis. One of the acute cases showed a positive reaction to this reaction test within one month after the infection. In the animal experiment the reaction changed to be positive as early as on the first week after the infection.

\section{REFERENCES}

1. Окаве, K., Koga, Y., Shibue, H. and Matsuse, M., : Immunological studies on schistosomiasis. 3. Intradermal and precipitin tests for schistosomiasis japonica. Kurume Med. J., Vol. 1, No. 2, 85-89. 1954.

2. OKABE, K. AND Tanaka, T., : A new urine presipitin reaction for schistosomiasis japonica, a preliminary report, Kurume Med. J., Vol. 5, No. 2, 45-52. 1958.

3. OKabe, K., Shi momura, M. and Tanaka, T., : Treatment of schistosomiasis japonica with antimony-a, a'-dimercapto-potassium succinate (TWSb) and follow-up by the urine and serum precipitin reactions. J. Kurume Med. Assoc., Vol. 22, No. 4, 1418-1424. 1959.

4. Окаве, K., Tanaka, T. and Umeno, N., : Immunological studies on acute and subacute schistosomiasis japonica. J. Kurume Med. Assoc., Vol. 23, No. 5, 1928-1930. 1960.

5. TANAKA, T., : Immunological studies on schistosomiasis japonica. J. Kurume Med. Assoc., Vol. 23, No. 8, 3220-3244. 1960. 